\title{
Low-frequency excitations in glassy selenium: A comparison of neutron-scattering and molecular-dynamics results
}

\author{
M. García-Hernández and F. J. Bermejo \\ Instituto de Estructura de la Materia, Consejo Superior de Investigaciones Científicas, Serrano 123, E-28006 Madrid, Spain \\ B. Fåk \\ Centre d'Etudes Nucléaires Grenoble, Département de Recherche Fondamentale sur la Matière Condensée, \\ Service de Physique Statistique Magnétisme et Supraconductivité, \\ Laboratoire de Magnétisme et Diffraction Neutronique, 85X, 38041 Grenoble CEDEX, France \\ J. L. Martínez \\ Instituto de Ciencia de Materiales, Sede B, Facultad de Ciencias, C-IV, Universidad Autónoma de Madrid, \\ Cantoblanco, E-28049, Spain \\ E. Enciso and N. G. Almarza \\ Departamento de Química-Física I, Universidad Complutense, Madrid, E-28040, Spain \\ A. Criado \\ Departamento de Física de la Materia Condensada, Universidad de Sevilla, Box 1065, Sevilla E-41080, Spain
}

(Received 29 December 1992; revised manuscript received 8 March 1993)

\begin{abstract}
The microscopic low-frequency dynamics of glassy selenium is investigated by means of the concurrent use of neutron inelastic scattering and computer simulations. A separation of the dynamic response in terms of intra- and interchain processes is achieved from the analysis of the simulation results. The $S(Q, E)$ dynamic structure factors are analyzed in terms of the frequency moments or from a model scattering law, and the wave-vector dependence of the relevant quantities is established. Finally, the anomalous behavior of the heat capacity at moderately low temperatures is shown to be originated by mostly interchain interactions.
\end{abstract}

\section{INTRODUCTION}

The interest in studies on the thermal and transport properties of glassy selenium stems from its technological importance in semi- or photoconducting devices, as well as for being one of the best-studied materials where the universal anomalies that characterize the glassy state have been studied in detail. ${ }^{1}$ However, the complicated atomic entanglements present in the liquid and glassy phases have precluded any microscopic analysis on the origin of the dynamic anomalies found by experimental means. ${ }^{2}$ In this respect, some of the ideas advanced more than a decade ago by Lucovsky and others ${ }^{3}$ on the origin of the different dynamical behavior in glassy and polycrystalline selenium remain to be assessed. This situation contrasts with the wealth of experimental data measured either in the hydrodynamic limit or using microscopic probes such as neutron scattering but actually analyzed in phenomenological or mesoscopic terms. ${ }^{4}$

Although a number of studies regarding the detailed dynamics of model glassy solids at microscopic scales have appeared, ${ }^{5,6}$ most of the published results concern computer (Lennard-Jones) glasses, which by their very nature lack any physical realization. On the other hand, an approach based upon the concurrent use of inelastic neutron scattering (INS), computer molecular-dynamics (MD) simulations for the glass and liquid phases, as well as lattice dynamics (LD) calculations for the polycrystalline reference state has been successfully employed for the assignment of the dynamic anomalies observed by INS (Ref. 7) and calorimetric means ${ }^{8}$ in real glasses composed by small molecular units. The comparison between experiment and computer simulations enables one on the one hand to assess the quality of the interparticle, effective potential used in the calculation, and, on the other hand it provides access to magnitudes hardly amenable to experimental measurement. From comparison between the LD results calculated for a polycrystal with experimental and MD data, a correlation between the excitations characteristic of the harmonic solid and those observed in the glass phase can be established.

The aims of the present work are twofold. First, to illustrate how such an approach can be used to analyze the finite-frequency features in the $S(Q, E)$ dynamic structure factor, and in particular to separate the lowfrequency contributions into those arising from individual chain (torsional) motions, and those originated from interchain interactions. Such a distinction is indispensable since a proper account of the very often complicated internal dynamics of the glass-forming entities appears as a prerequisite before a fundamental understanding on the universal origin of the low-frequency anomalies in glasses of widely varying chemical composition can be achieved. On the other hand, and up to the authors' knowledge, 
this work constitutes the first attempt to study in some detail the vibrational (i.e., short-time) dynamics of glassy selenium at length and time scales compatible with INS and computer simulations. For such a purpose, experimental data which, by force, are measured under adverse conditions (i.e., low resolution in energy transfers and spanning a limited extent in wave vectors) are compared with lattice and molecular-dynamics calculations carried out using the same model potential for the polycrystalline and glass phases, respectively. Because there is no available dynamical model of general validity applicable for the analysis of the experimental spectra, our approach follows two different routes, such as the analysis of the spectra in conventional terms using damped harmonic oscillator functions and a model-free approach based on the frequency moments calculated by integration of the measured intensities, and the results derived from both are then compared.

Some previous results concerning the employed model potential for a study of the liquid phase have already been given, ${ }^{9}$ as well as a preliminary brief account ${ }^{10}$ of results for the glass, so that only the very relevant experimental and calculational details are given in the next paragraphs.

\section{EXPERIMENTAL AND COMPUTATIONAL DETAILS}

Although a number of INS experiments have been reported in the literature, ${ }^{11}$ their main emphasis was the measurement of the $Z(E)$ generalized vibrational density of states using high resolution in energy transfers, and therefore a very limited dynamic range at constant momentum transfer was accessible, especially for $Q$ values below the first diffraction peak. Our aim is thus to provide some data regarding the inelastic intensities at relatively low wave vectors, using higher incident energies than those previously employed, something which obviously leads to low resolution in energy transfers.

The experiments were carried out using the DN1 tripleaxis spectrometer at the Siloë reactor, CEN Grenoble (France), and the experimental conditions are described in Ref. 10. A sample of corrected spectra which have been normalized to the static structure factor is shown in Fig. 1 on a logarithmic scale, used to avoid the truncation of the elastic peak intensity.

The MD calculations were carried out under conditions similar to those already reported. ${ }^{9,10}$ The simulation box contained 32 chains of 20 atoms each and the interaction potential corresponded to the one already referred. ${ }^{9}$ No account for three-fold defects was made because of the difficulty of optimizing the parameters required to construct a strong directional-bonding algorithm compatible with what is known from first-principles simulations. ${ }^{12}$ Relevant details concerning the validation of the model potential are given in Ref. 10.

The intermediate scattering functions corresponding to a chain of selenium atoms were calculated following
$F_{\mathrm{ch}}(Q, t)=\frac{1}{N_{\mathrm{at}}} \sum_{i, j=1}^{N_{\mathrm{at}}}\left\langle\exp \left[i \mathbf{Q} \cdot \mathbf{r}_{j}(t)\right] \exp \left[-i \mathbf{Q} \cdot \mathbf{r}_{i}(0)\right]\right\rangle$

where $\mathbf{r}_{i}$ is the relative displacement of atom $i$ th relative to the center of mass (c.m.) of the chain and $N_{\text {at }}$ the number of atoms per chain. The total atom-atom function was calculated from

$$
F(Q, t)=\frac{1}{N} \sum_{i, j}\left\langle\exp \left[i \mathbf{Q} \cdot \mathbf{a}_{i}(t)\right] \exp \left[-i \mathbf{Q} \cdot \mathbf{a}_{j}(0)\right]\right\rangle
$$
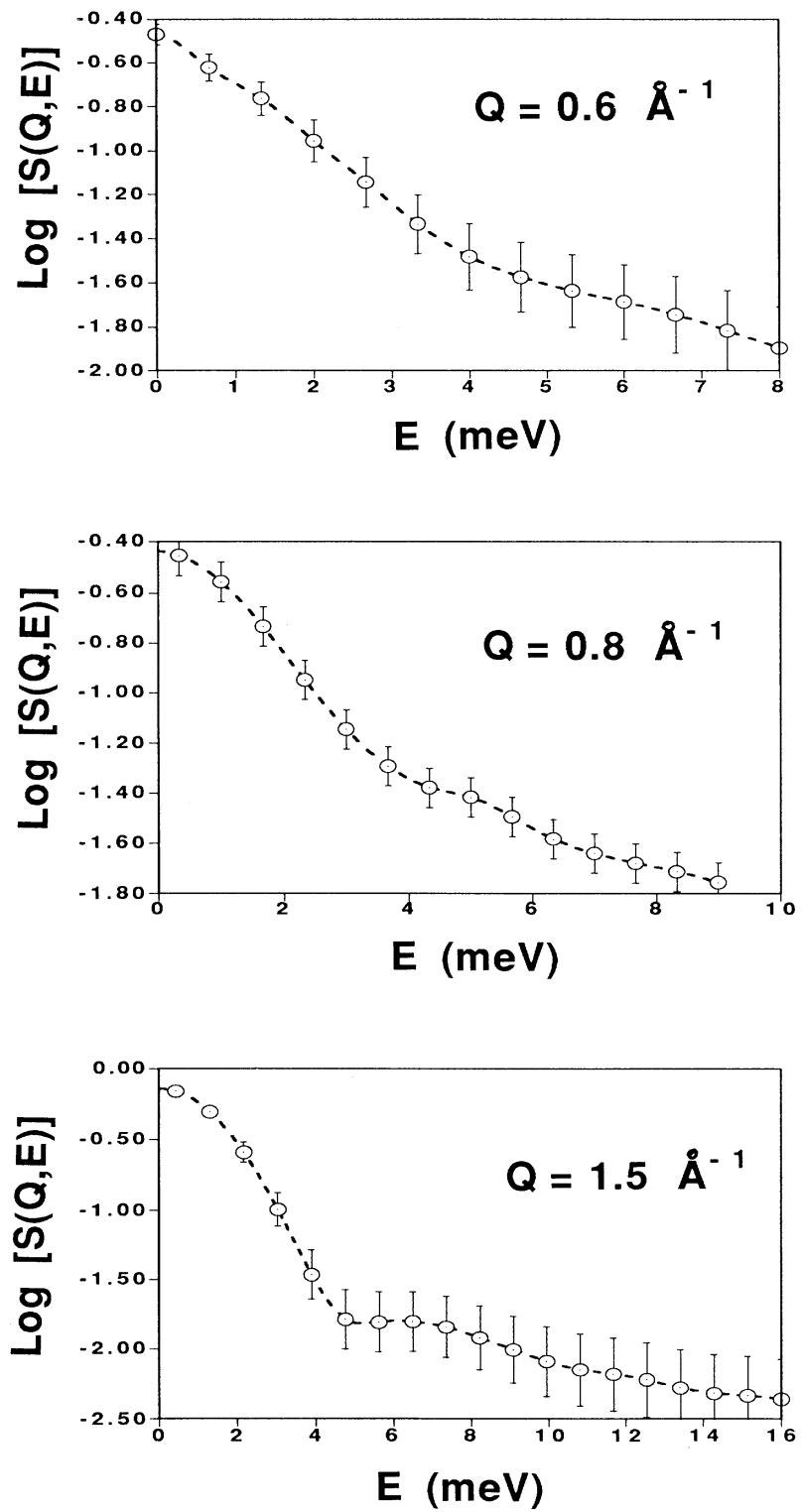

FIG. 1. Corrected inelastic intensities corresponding to the energy-loss side of the experimental spectra. The logarithm is to base 10 . 
where $\mathbf{a}_{i}$ now stands for the position of an individual atom and $N$ is the total number of atoms in the simulation box. The static, $t=0$ limit of this function leads to the $S(Q)$ structure factor whereas the persistent part of it, taken at $t=\infty$, gives $S(Q, 0)$. In order to alleviate the truncation problems in the subsequent Fourier transformation into frequency space, the residual elastic intensity [i.e., $F(Q, t=\infty)$ ] was subtracted using for such a purpose the values of the intermediate scattering functions taken at $10 \mathrm{ps}^{13}$. On the other hand, as will be discussed below, several functions related to the zero and infinitetime limits of the intermediate scattering functions have been calculated. In particular the functions $h(Q)$ and $f(Q)$ defined as

$h(Q)=\frac{F(Q, t=\infty)}{F(Q, t=0)}=\frac{S(Q, 0)}{S(Q)}$

$f(Q)=\frac{F(Q, t=\infty)}{F(Q, t=0)-F(Q, t=\infty)}=\frac{S(Q, 0)}{S_{\text {inel }}(Q)}$,

where $S_{\text {inel }}(Q)$ represents the (energy-integrated) inelastic intensity, are of experimental and theoretical relevance within present-day kinetic theory approaches ${ }^{14}$ since they provide detailed information about the wavevector dependence of an average Debye-Waller factor (i.e., containing contributions arising from all the dynamical processes), and can be contrasted with predictions now available. In what follows the subscript ch will denote quantities that refer to an individual chain, whereas the unsubscripted functions will refer to the total atomatom magnitudes. The velocity correlation functions corresponding to c.m., an individual chain, and the total atomic function were also calculated.

Although a valence force field has been developed to account for the detailed dynamics of trigonal selenium, 15 its transferability for the glass and liquid phases is questionable due to the fact that interchain bonding is substantially weaker in the disordered states. ${ }^{3}$ In order to test the reliability of the potential model used in this work, the structure and thermodynamics of the glass and trigonal polycrystalline phases were compared with experimental measurements. In particular the calculated $S(Q)$ static structure factor, the $g(r)$ radial distribution function for the glass, and the specific heat for both glass and the polycrystal were compared with experimental measurements, ${ }^{10}$ showing a good agreement for the structural properties, and tolerable agreement for the specific heat. For the polycrystal, a LD calculation was carried out for a unit cell representative of trigonal selenium. The methods used for the calculation which is based upon the molecular Born-von Karman framework as well for the orientational averaging and the computation of the neutron-scattering functions have been described elsewhere. ${ }^{7}$

The dynamics of such a complicated solid can only be analyzed in descriptive terms by means of average quantities such as mean frequencies and damping factors. Furthermore, due to the low resolution of the experimental measurements, a direct analysis of the measured intensities in terms of some prespecified model function will not lead to significant results unless a number of constraints are introduced to bound the model parameters. To proceed, we have used the $S(Q, E)$ dynamic structure factors calculated from the MD simulation as a starting point in order to investigate the minimum requirements for such a model, so that it could be used afterwards for the analysis of the experimental spectra.

For frequencies below $20 \mathrm{meV}(\approx 5 \mathrm{THz})$ and within the momentum transfers $0.3 \AA^{-1} \leq Q \leq 2.0 \AA^{-1}$, a model scattering law given in terms of a $\delta$ function of small amplitude to account for the residual elastic intensity [i.e., due to the incomplete subtraction of the $F(Q, t=\infty)$ pedestal] and two damped harmonic oscillators $^{16}$ well separated in frequency, was found to be adequate to reproduce the inelastic intensity of the calculated MD spectra. Such a model scattering law will then read as

$$
\begin{aligned}
I_{\text {model }}(Q, E) & =\exp [-2 W(Q)]\left[S(Q) \delta(E=0)+S_{1}\left(E_{1}, \Gamma_{1}\right)+S_{2}\left(E_{2}, \Gamma_{2}\right)\right] \otimes R(E) \\
S_{i}\left(E_{i}, \Gamma_{i}\right) & =n(E) C_{i}(Q) \frac{4 E \hbar \omega_{i} \Gamma_{i}}{\left(E^{2}-E_{i}^{2}\right)^{2}+4 E^{2} \Gamma_{i}^{2}} \\
E_{i}^{2} & =\left(\hbar \omega_{i}\right)^{2}+\Gamma_{i}^{2}
\end{aligned}
$$

where the exponential contains an average Debye-Waller factor, the two oscillator functions are implicitly assumed to be energy and wave-vector dependent, $R(E)$ accounts for the finite resolution in energy transfers due to the time truncation of the calculated $F(Q, t)$ intermediate scattering functions $(\approx 10 \mathrm{ps})$, and the quantities specifying the frequency and damping are implicitly assumed to be wave-vector dependent. The two functions are specified by the $C_{i}(Q)$ line-strength factor, the $\omega_{i}$ bare excitation frequency, and the $\Gamma_{i}$ damping factor. The term $n(E)$ represents a detailed balance factor, and the model is then convoluted with the $R(E)$ resolution function which accounts for the effect of truncation at finite times. An additional oscillator function is required to account for
$Q$ values above $Q_{p}$ [i.e., the momentum transfer corresponding to the maximum of $S(Q)$ ], due to the increasing weight of the high-frequency tails of the spectra, something which substantially complicates the obtainment of reliable estimates for the parameters above this $Q$ value.

Some of the calculated MD spectra as well as their approximations in terms of the equations given above are shown in Fig. 2, where it can be seen that such a relatively simple approximation gives an adequate account of the inelastic intensities for the specified range of frequencies. The most remarkable characteristics of the MD spectra for the referred range of wave vectors are the presence of a finite-frequency peak of relatively small intensity located at about $2 \mathrm{meV}$ and the strong, broad 
background of excitations which extends up to about 20 $\mathrm{meV}$. The experimental spectra were also analyzed on the same terms, and the relevant frequency and damping parameters will be discussed below.

\section{RESULTS}

\section{A. Generalized frequency distributions and heat capacity}

The $Z(E)$ generalized vibrational density of states for glassy selenium has been estimated from experimental means by several authors ${ }^{11}$ by means of high-resolution
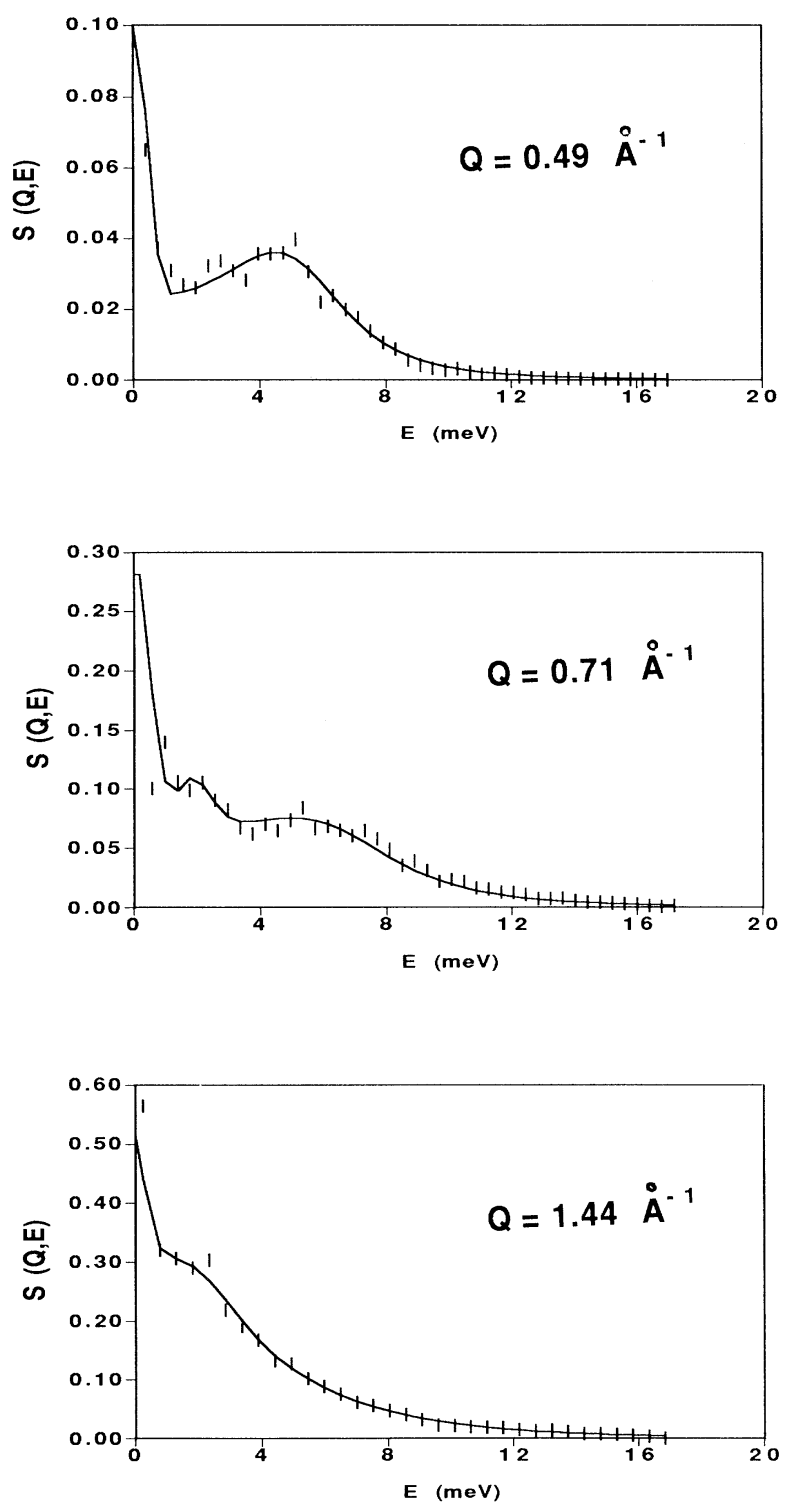

FIG. 2. A comparison between $S(Q, E)$ dynamic structure factors calculated from the MD simulation results (vertical bars) and the fitted model using Eqs. (5)-(7) (solid lines).
INS. Although the general shape of these curves agrees in all cases some systematic differences remain regarding the low-frequency tails.

Even if an accurate calculation of the generalized vibrational frequency distribution $Z(E)$ cannot be reliably accomplished with the present set of experimental data, an estimate of $Z(E)$ was obtained from the quantities $J_{l}(Q, E)=\left(E^{2} / Q^{2}\right) S(Q, E)$, which were evaluated from fits to the experimental spectra as described above, and were subsequently corrected for the Debye-Waller term, for which the value derived for the mean square displacement from the simulation was taken, the structure factor and Bose factor, following the prescription of Buchenau, ${ }^{17}$ and the result is compared with the simulation results as well as with previous experimental data in Fig. 3(a). Some systematic differences between the low-frequency tail of Phillips et $a l^{2}{ }^{2}$ and that calculated from the MD and present experimental results are noticeable. However, the relatively low-energy resolution of the present experiment as well as possible inaccuracies of the used potential at low frequencies hinder any more detailed comparison. The most remarkable feature that arises from such a decomposition of the $Z(E)$ in terms of the c.m. and total functions lies in the close resemblance of the spectra calculated within the incoherent approximation from the c.m. component, with the inelastic feature observed in the experimental spectra measured in higher-resolution experiments as it is illustrated in Fig. $3(\mathrm{a})$.

The shape of the total $Z(E)$ function agrees in general terms with those derived from experimental means, ${ }^{11}$ the presence of a double peak structure in the bondstretching contour being one of the most noticeable differences. It is difficult to perform a quantitative comparison between experiment and calculation since resolution effects at these relatively large energy transfers become important in time-of-flight spectroscopy. In any case, it is worth noting that both experiment and calculation show approximately the same width for this spectral band. The maximum of the higher energy peak on the other hand matches the position of that reported by Gompf. ${ }^{11}$

To provide an indication of the contributions arising from different atomic motions to the total amplitude entering the Debye-Waller factor, the integral over $Z(E)$ (see below) was evaluated using as upper limits the value of $6 \mathrm{meV}$ which bounds all the acoustic and the lower lying optic modes, $10 \mathrm{meV}$ which is the boundary of the first prominent band, and $18 \mathrm{meV}$ which marks the gap edge. The values of the integral in percentage units of the value calculated using the whole dynamic range were 91.5, 96.9, and 99.3, respectively. Such a result indicates the small contribution of the stretching modes to the mean squared displacement (about $0.7 \%$ ) as well as the rather moderate contribution of the bending modes (a bit less than 5\%), whereas acoustic and torsional vibrations account for up to $97 \%$ of the observed displacement.

The fluctuations of the configurational energy were monitored since the mean square value of this quantity provides a route for the computation of fundamental thermodynamic functions, alternative to those based 
upon the frequency spectrum. ${ }^{18}$ The total heat capacity is then expressed (in units of the gas constant $R$ ) as the sum

$$
\frac{C_{v}}{R}=1.5+\left[\frac{N_{\mathrm{mol}}}{N_{\mathrm{at}}} \frac{1}{T_{\mathrm{red}}^{2}} \sum_{i} \sum_{j}\left(U^{2}-\bar{U}^{2}\right)_{i j}\right]
$$

where $N_{\text {mol }}$ and $N_{\text {at }}$ are the number of chains in the sim-
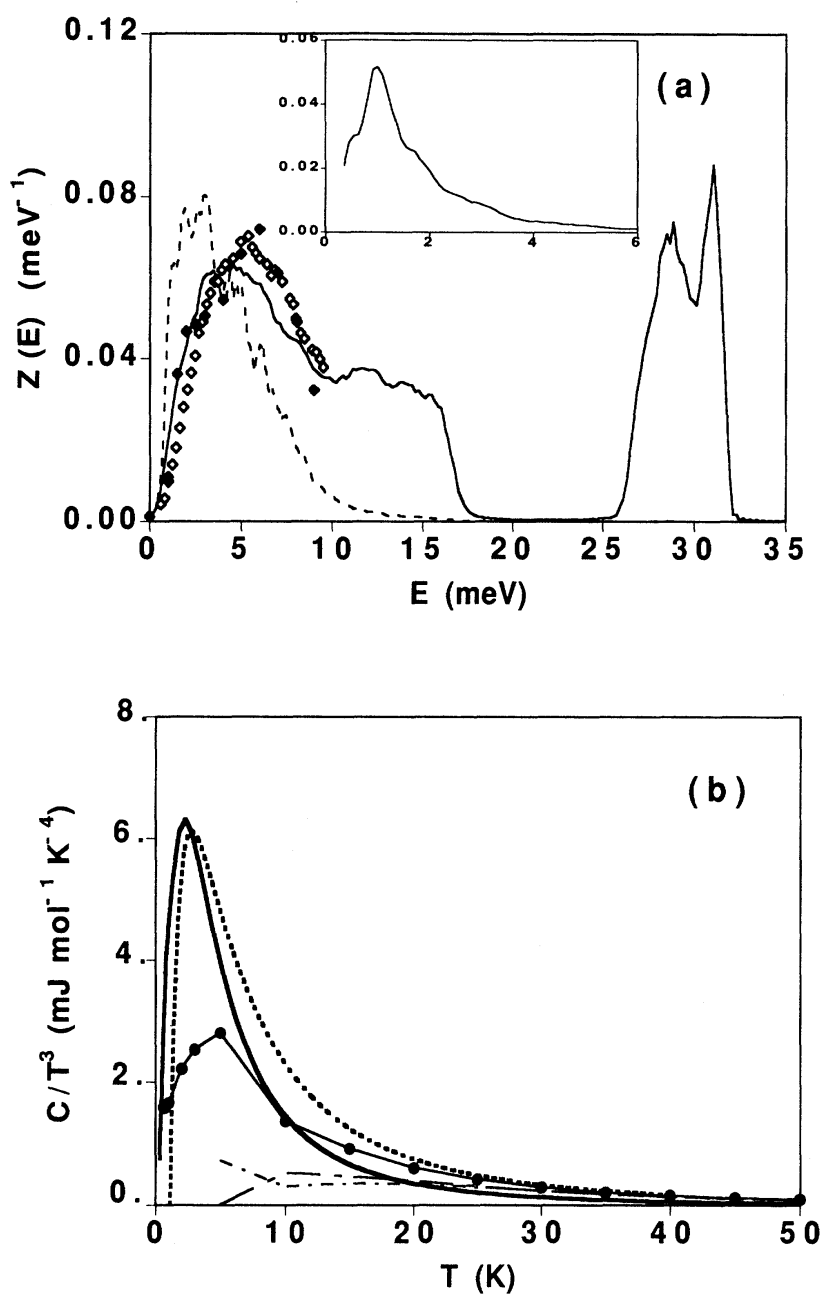

FIG. 3. (a) The experimental $Z(E)$ for glassy Se at the same thermodynamic state from a higher-energy resolution experiment (Ref. 2) is shown by the open lozenges. The solid lozenges represent the estimate for $Z(E)$ derived from the present set of measurements. The calculated total function for the glass is shown by the solid line, and the c.m. component by the dashed line. The inset shows the resulting $Z(E) / E^{2}$ function [i.e., $S(Q, E)$ in the incoherent approximation], as calculated from the c.m. function. (b) A comparison of the experimental and calculated $C(T) / T^{3}$ functions. The thick solid line corresponds to that calculated from the c.m. $Z(E)$ function and that calculated from the total $Z(E)$ is shown by the dotted line. The experimental data from Refs. 19 and 27 for the glass are shown as filled circles with a solid line. The two lines at the bottom (dashed and dot-dashed) are experimental measurements for trigonal selenium (Ref. 19). ulation box and the number of atoms per chain, respectively, $T_{\text {red }}=0.5$ and the subindexes run over the five contributions to the total energy $U$ and its average value $\bar{U}$ [inter- and intrachain van der Waals (vdW), bond torsions, bond stretchings, and bond-angle bendings], thus leading to a sum of 25 contributions. Such an analysis of the energy fluctuations yielded an array of coupling coefficients (i.e., contributions to the configurational heat capacity from each one of the coordinates used to express the potential as well as from interactions between them) which is shown in Table I. The diagonalization of such an array thus provides some indications regarding the principal components of the coupling coefficients matrix. From the analysis of the eigenvalues and their corresponding eigenvectors, the following qualifications can be made.

(1) The five coordinates used to express the total potential are strongly coupled. As an example, the coupling coefficients (normalized to one) between bond bending and bond stretching, or bond torsion and intrachain vdW amount to -0.96 and -0.94 , respectively. (2) The relative contribution of the interchain vdW to the total heat capacity amounts to $41 \%$ at the temperature under consideration. The coupling of this coordinate to the other degrees of freedom is evenly distributed with coefficients not exceeding, in absolute values, -0.29 or below -0.15 .

(3) The relative weight of the interchain vdW contribution can then be used as a scale factor so that the total heat capacity as well as the c.m. contribution to it can be evaluated from the corresponding $Z(E)$ functions. As shown in Fig. 3(b), both the total $Z(E)$ and the c.m. function give rise to $C(T) / T^{3}$ curves, showing a well-defined maximum at a temperature which is in agreement with the experimental values. ${ }^{19} \mathrm{~A}$ noticeable excess is clearly seen in Fig. 3(b) with respect to the experimental value, which may be a consequence of the inability of the model potential to deal with very lowfrequency excitations. On the other hand, and as can easily be seen from the graph, the c.m. contribution to the total $C(T)$ becomes the relevant one at temperatures below $10 \mathrm{~K}$.

The value for the total configurational heat capacity $18.9 \mathrm{~J} \mathrm{~K}^{-1} \mathrm{~mol}^{-1}$ calculated in such a way compares favorably with the experimental measurement for this temperature of $18.255 \mathrm{~J} \mathrm{~K}^{-1} \mathrm{~mol}^{-1} .^{19} \mathrm{On}$ the other hand the value estimated from integration of the $Z(E)$ function gives an estimate of $18.97 \mathrm{~J} \mathrm{~K}^{-1} \mathrm{~mol}^{-1}$ for this magnitude at $T=100 \mathrm{~K}$, and such a small difference between both values gives an indication of the importance of anharmonic effects at this thermodynamic state. As a matter of fact, from such agreement it seems clear that a quasiharmonic approximation for the calculation of the thermodynamic functions from the frequency spectrum calculated for this temperature is entirely justified.

In summary, the total heat capacity as calculated from the energy fluctuations or from integration of $Z(E)$ at $T=100 \mathrm{~K}$ for the glass is acceptably reproduced using the adopted model potential, and, on qualitative grounds, the range of validity of such a prediction can be extended down to a few degrees. It is not entirely clear if the origin of the excess heat capacity at about 5 
TABLE I. Calculated contributions from inter- (vdW inter) and intrachain (vdW intra) van der Waals interactions, bond stretch (Str.), bending (Bend.), and torsional (Tors.) vibrations to the total configurational heat capacity from the different terms entering the effective interparticle potential. Values are given in $R$ units.

\begin{tabular}{|c|c|c|c|c|c|}
\hline Coord. & $\mathrm{vdW}_{\text {inter }}$ & vdW intra & Str. & Bend. & Tors. \\
\hline $\mathrm{vdW}_{\text {inter }}$ & 0.9235 & -0.1865 & -0.1727 & -0.1523 & -0.1354 \\
\hline$v W_{\text {intra }}$ & & 0.2123 & -0.0261 & 0.0046 & 0.0025 \\
\hline Str. & & & 0.6231 & -0.1824 & -0.0089 \\
\hline Bend. & & & & 0.5800 & -0.0605 \\
\hline Tors. & & & & & 0.2436 \\
\hline
\end{tabular}

$\mathrm{K}$ is an artifact of the model potential or whether it arises from some other effects such as system-size limitations.

\section{B. Wave-vector-dependent response}

\section{Debye-Waller factors}

The $S(Q, 0)$ and $S(Q)$ strictly elastic and the total structure factors as calculated from the experimental spectra are shown in Fig. 4 and are in good agreement with results obtained from diffraction means ${ }^{10}$ as well as from those derived from high-resolution INS, ${ }^{2}$ although in the latter case both functions become identical below $1 \AA^{-1}$ due to the limited dynamic range used. An average Debye-Waller factor $\exp [-2 W(Q)]$ is therefore accessible from the ratio $S(Q, 0) / S(Q)$ of experimental intensities or from its simulation counterpart cast in the form of intermediate scattering functions. The quantities $F_{\mathrm{ch}}(Q, t=0), F_{\mathrm{ch}}(Q, t=\infty)$ as well as their correlates for the $F(Q, t)$ total atom-atom function are also shown in Fig. 4. The former quantities contain all the relevant information regarding the Debye-Waller factors for an individual chain, $F_{\mathrm{ch}}(Q, t=0)$ being a static structure factor (molecular form factor) which is dominated by the coherent response due to short-range correlations. The corresponding structure factors for the total intermediate scattering function are also given in the figure, and show a well-defined maximum at $Q$ values about that corresponding to the maximum of the $S(Q)$ static structure factor.

As mentioned in the previous sections, the quantities $h(Q), f(Q)$ have been calculated for both cases using Eqs. (3) and (4) from the above referred data and the results are shown in Fig. 5. The $h_{\mathrm{ch}}(Q)$ function shows, for this range of momentum transfers, a smooth decaying behavior which can be adequately approximated by a parabolic law such as $h_{\mathrm{ch}}(Q)=1-a Q^{2}$, or by a Gaussian $h_{\mathrm{ch}}(Q)=\exp \left(-Q^{2} a^{2}\right)$ giving values for the free parameter of $0.0212(6) \AA^{2}$ and $0.0237(2) \AA^{2}$, respectively. On the other hand, the $h(Q)$ which corresponds to the total function shows two well-defined peaks at $Q \approx 1.4$ $\AA^{-1}$ and $Q \approx 2 \AA^{-1}$, respectively. Some comments are in order regarding these two functions. First of all, the fact that the $h_{\mathrm{ch}}(Q)$ function follows an approximately parabolic dependence with momentum transfers can be rationalized as follows. At low $Q$ values the spectra are dominated by correlations between atoms separated by relatively large distances which cannot move in phase due to the force exerted by atoms belonging to neighboring chains. As the distances being sampled are smaller (i.e., larger momentum transfers), localized atomic motions become more evident, and their contributions are increasingly important for large- $Q$ values. Because of the large number of atomic pairs which contribute to this intensity and the fact that these quantities represent energy-integrated magnitudes, any phase relationship between waves scattered by such pairs is washed out, and therefore the inelastic intensity coming from individual chain motions is reminiscent of that corresponding to an average atom with an effective mass $M_{\text {eff }}$.

The shape of the $h_{\mathrm{ch}}(Q)$ curve closely resembles that of a Lamb-Mössbauer factor for incoherent scattering from a solid [i.e., $h_{s}(Q)=1-\left(r_{s} Q\right)^{2}+O\left(Q^{4}\right)$ ], or the corresponding quantity for a glass $h_{s}(Q)=\exp \left(-Q^{2} r_{s}^{2}\right)$, something which would allow the interpretation of the $a$ parameter given above as a mean square displacement. If such an identification is carried out, a value not far from the one reported in Refs. 4 and $20, \approx 0.025 \AA^{2}$ for $T=100 \mathrm{~K}$ which corresponds to an estimation from the experimental spectra, is found. Further support for this interpretation comes from the fact that $h_{\mathrm{ch}}(Q)$ approaches unity at low $Q$, contrary to the total function which is bounded below unity for all of the explored $Q$ range. As can also be seen from the graph, the presence of a double peak structure in the total $h(Q)$ function is also borne out from the experimental measurement where, on the other hand, it shows a more marked character, and further comments regarding this topic are deferred to the discussion section.

The value calculated for the mean atomic displacement can also be compared with that calculated from integration of the simulated $Z(E)$ density of states since

$$
\begin{gathered}
h_{\mathrm{ch}}(Q) \approx 1-\left(r_{s} Q\right)^{2} \approx 1-\frac{1}{6} Q^{2} u^{2}, \\
u^{2} \approx \frac{3 k_{B} T}{M_{\mathrm{eff}}} \int_{0}^{E_{\max }} Z(E) E^{-2} d E,
\end{gathered}
$$

where $E_{\max }$ is a cutoff energy which for the present computation was set to accommodate the whole spectral range $(\approx 40 \mathrm{meV})$. From such a comparison a value for the effective mass $M_{\text {eff }}$ of 2.48 times the mass of a selenium atom was derived.

It is obviously difficult to derive an accurate value for the mean square displacements corresponding to the total atomic function, since the oscillations in $h(Q)$ which arise 
from interference effects will lead to the wave-vectordependent quantity $r_{s}^{2}(Q)=6 W(Q) / Q^{2}$. An average over a region of momentum transfers in order to remove the oscillatory behavior has been performed by Buchenau et $a l .,{ }^{4,20}$ and the value quoted above, very close to the one derived from an individual chain, was obtained.

\section{Finite-frequency response}

A set of spectra as calculated from the polycrystalline average of $S(Q, E)$ calculated from LD as well as their
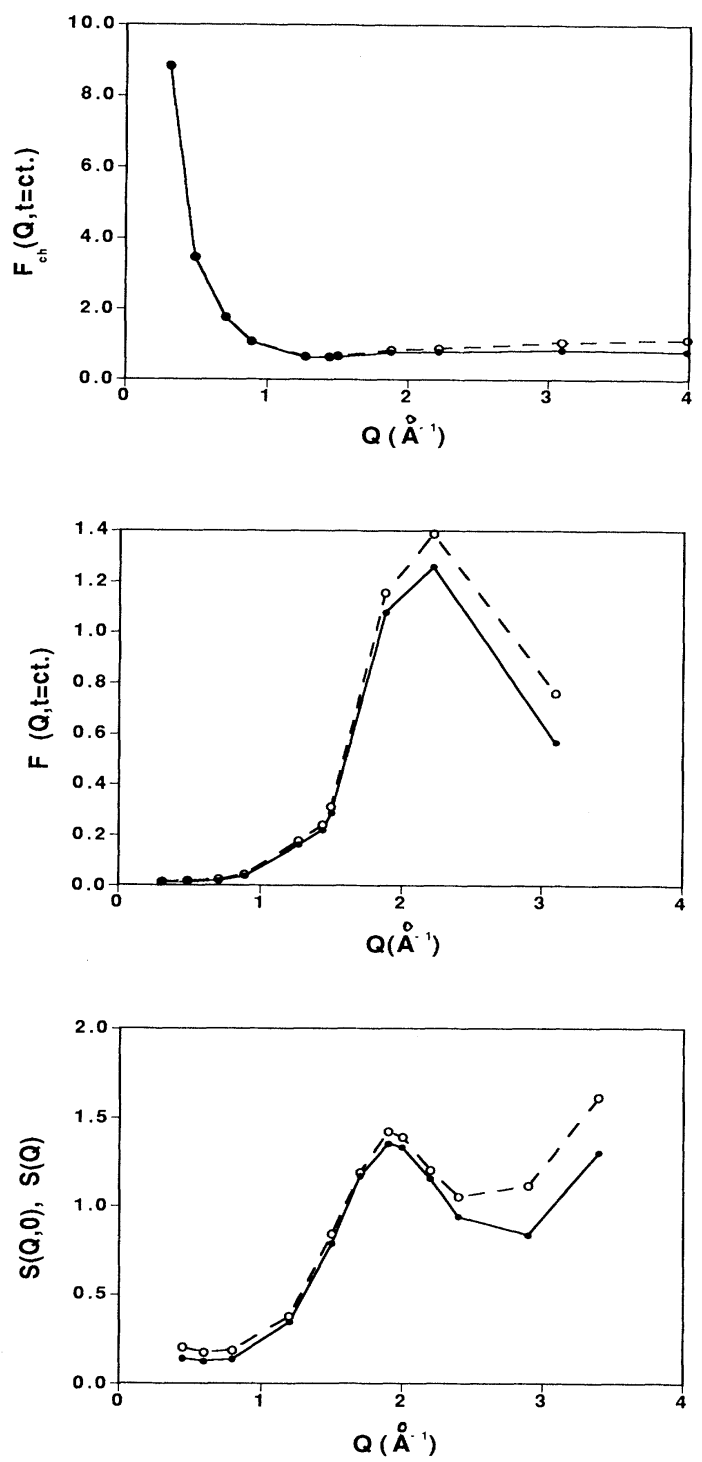

FIG. 4. The upper frame shows the limiting values for the intermediate scattering functions for an individual chain of selenium atoms. Open circles with a dashed line represent the $F(Q, t=0)$ function and solid circles with solid line the $F(Q, t=\infty)$ counterpart. The middle frame shows the corresponding function for the total spectra. The same symbols are used. The lower frame depicts the experimental data. Here open circles stand for $S(Q, 0)$ and filled circles show the total $S(Q)$.
MD counterparts for the glass are shown in Fig. 6. The quantity plotted in such graphs corresponds to the $J_{l}(Q, E)$ longitudinal current correlation function in order to circumvent the residual elastic scattering. The first maxima of these functions $\omega_{m}(Q)$ is frequently used as an average frequency in order to describe the wave-vector dependence of the vibrational collective dynamics. ${ }^{21}$ As is clearly seen from the graph, both the crystal and glass spectra show two well-defined frequency regions separated by a gap. The assignment of the individual excitation frequencies in the single-crystal case is now well established $^{1}$ so that the interest of the present exercise lies in the detailed (i.e., $Q$-dependent) comparison of the polycrystalline and glass spectra. At the lowest wave vector where such a comparison is feasible, $Q \approx 0.3 \AA^{-1}$, the LD spectra is basically composed of the manifold of acoustical peaks and some relatively small peaks arising from the higher lying optic branches. According to the currently accepted assignment, the excitations appearing at frequencies below $6 \mathrm{meV}$ either belong to the acous-
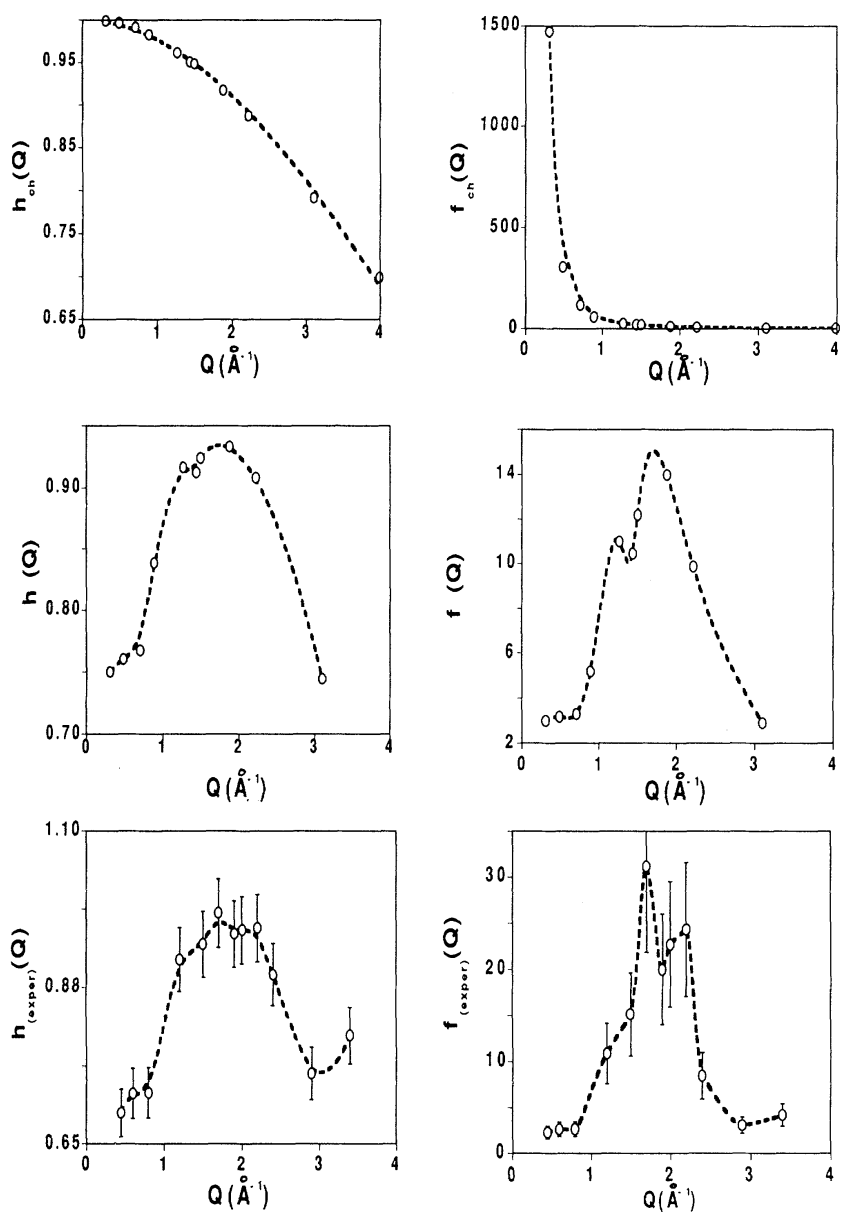

FIG. 5. The left-hand side of the figure shows the DebyeWaller factors [i.e., $h(Q)$ functions] for an individual chain (top), the total simulated spectra (middle), and present experimental results (bottom). The right-hand side shows the corresponding ratios of the total elastic to inelastic intensities [i.e., $f(Q)$ functions]. 
tical branches or to some low-frequency optic modes, es-

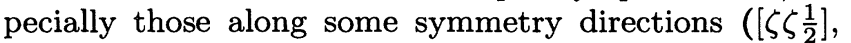
$\left[\frac{1}{3} \frac{1}{3} \zeta\right]$, and [ $\left.\zeta \zeta 0\right]$ Ref. 1) some of which show phonon frequencies as low as $3.35 \mathrm{meV}$ [the point for $\left(\frac{1}{3}, \frac{1}{3}, 0\right)$ at $\mathrm{T}=300 \mathrm{~K}$ ]. Between $6 \mathrm{meV}$ and the gap edge $(\approx 16$ $\mathrm{meV}$ ), most of the intensity arises from excitations involving internal modes of the selenium chains such as bond torsions, bond bendings, and interactions between them. The bond strectch and some of the modes related to it are located within the $27 \leq E \leq 32 \mathrm{meV}$ frequency range, and show an intensity which becomes maximal for $Q$ values around the fundamental Bragg peak $(\approx 1.8$ $\AA^{-1}$ ) as well as a negligible frequency dispersion. On the other hand, although at this low- $Q$ value the shape of the glass spectra is clearly a remnant of the polycrystal, the intensity ratio between the two more intense peaks is far different from that characteristic of the crystal. The difference in the overall shape of the spectra corresponding to the two states becomes more pronounced as $Q$ is
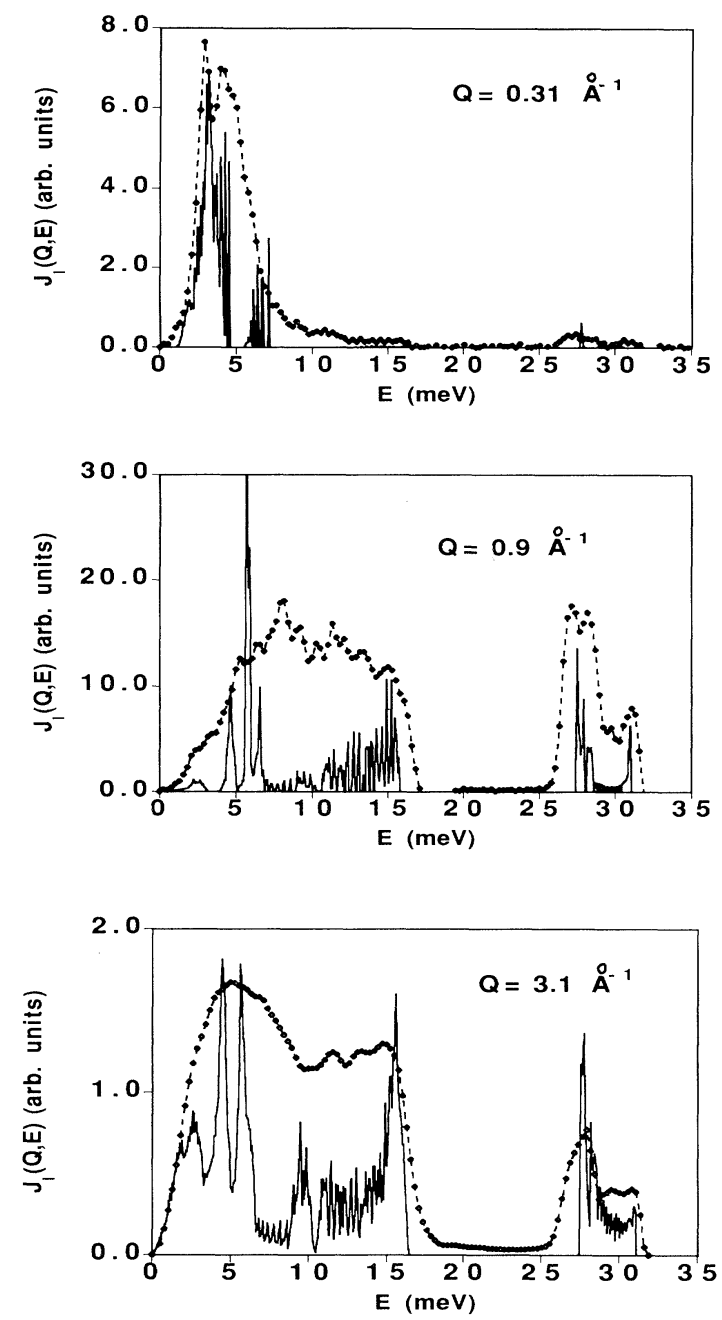

FIG. 6. $\quad J_{l}(Q, E)$ longitudinal current correlation functions as calculated from the LD (solid lines) for the polycrystal and MD (filled lozenges and dashes) results for the glass. The corresponding values of momentum transfers are given in the insets. increased up until that characteristic of an average Brillouin zone boundary in the crystal $\left(\approx 0.9 \AA^{-1}\right)$. Above such a value, the shape of both sets of spectra becomes closer again.

As mentioned above, the $\omega_{m}(Q)$ frequencies can be used to provide an approximate measure of the dispersion behavior. For the polycrystal, the presence of a clear, intense peak up to wave vectors about $1 \AA^{-1}$ enables one to plot such a quantity unambiguously. Beyond such momentum transfers the appearance of a large number of excitation peaks hinders the continuation to larger- $Q$ values. The situation is relatively easier in the glass case since only the frequencies corresponding to the maxima of the first broadband are taken into consideration. A plot of such magnitudes as well as that corresponding to the experimental measurement is shown in Fig. 7 alongside with the hydrodynamic limits for the sound frequencies as calculated from the $c_{D}$ Debye velocities. The latter quantities, used to calculate the linear laws for the crystal, were those given by Meissner ${ }^{19}\left(c_{D}=1727 \mathrm{~ms}^{-1}\right.$ for the $T=0 \mathrm{~K}$ solid), and that corresponding to the glass was taken from data given in Ref. $4\left(c_{D}=1170 \mathrm{~ms}^{-1}\right)$.

The crystal data show a strikingly smooth behavior up to $1 \AA^{-1}$. In the long-wavelength limit, the straight line depicting the $c_{D} Q$ hydrodynamic sound and $\omega_{m}(Q)$ for the polycrystal basically coincide up to wave vectors about $Q \doteq 0.3 \AA^{-1}$, something which allows the identification of such a peak in the polycrystalline spectra as arising from soundwave excitations. The phase and group velocity dispersions, also shown in the graph, give additional support to the assignment of such a peak. A maximum of the phase velocity dispersion is clearly seen in the glass (i.e., "positive dispersion" in hydrodynamical terms) whereas the crystal data linearly extrapolates to the hydrodynamic limit. The presence of such maxima in the curve corresponding to the glass can be easily explained if the increasing weight of the opticlike modes as the wave vector is increased towards $Q_{p} / 2$ is accounted for. It is also interesting to note the difference between the momentum-transfer value corresponding to zero group velocity in the polycrystal and glass which, in the first case, coincides with the zone boundary, and may serve to indicate the existence of a pseudozone boundary in the glass in a dynamical sense.

On the other hand, the $\omega_{m}(Q)$ curve corresponding to the MD data shows, for momentum transfers above $Q=0.49 \AA^{-1}$, excitation energies high above those corresponding to the hydrodynamic limit and at $Q=1 \AA^{-1}$ is shifted towards frequencies which are far higher than what could, in principle, be expected, since both real and simulated glasses behave elastically as softer bodies than the crystal. ${ }^{19}$ However, from an inspection of the graph showing the phase velocities for both states, it is clear that, although both curves approach the hydrodynamic limit correctly, the presence of optic-like excitations in the glass at low- $Q$ values is far more important than in its crystalline correlate.

In order to describe the wave-vector-dependent frequency distributions without recourse of any particular model to represent the line shape, the first $\mu_{n}(Q)$ reduced frequency moments computed as frequency integrals over 
$S(Q, E)$ were calculated in a previous work and compared with the relevant hydrodynamic limits. ${ }^{10}$ Our aim here is to provide a comparison between the frequency moments of the $S_{\mathrm{ch}}(Q, E)$ dynamic structure factor associated with the single-chain dynamic response and those calculated from the total atom-atom function. Such a
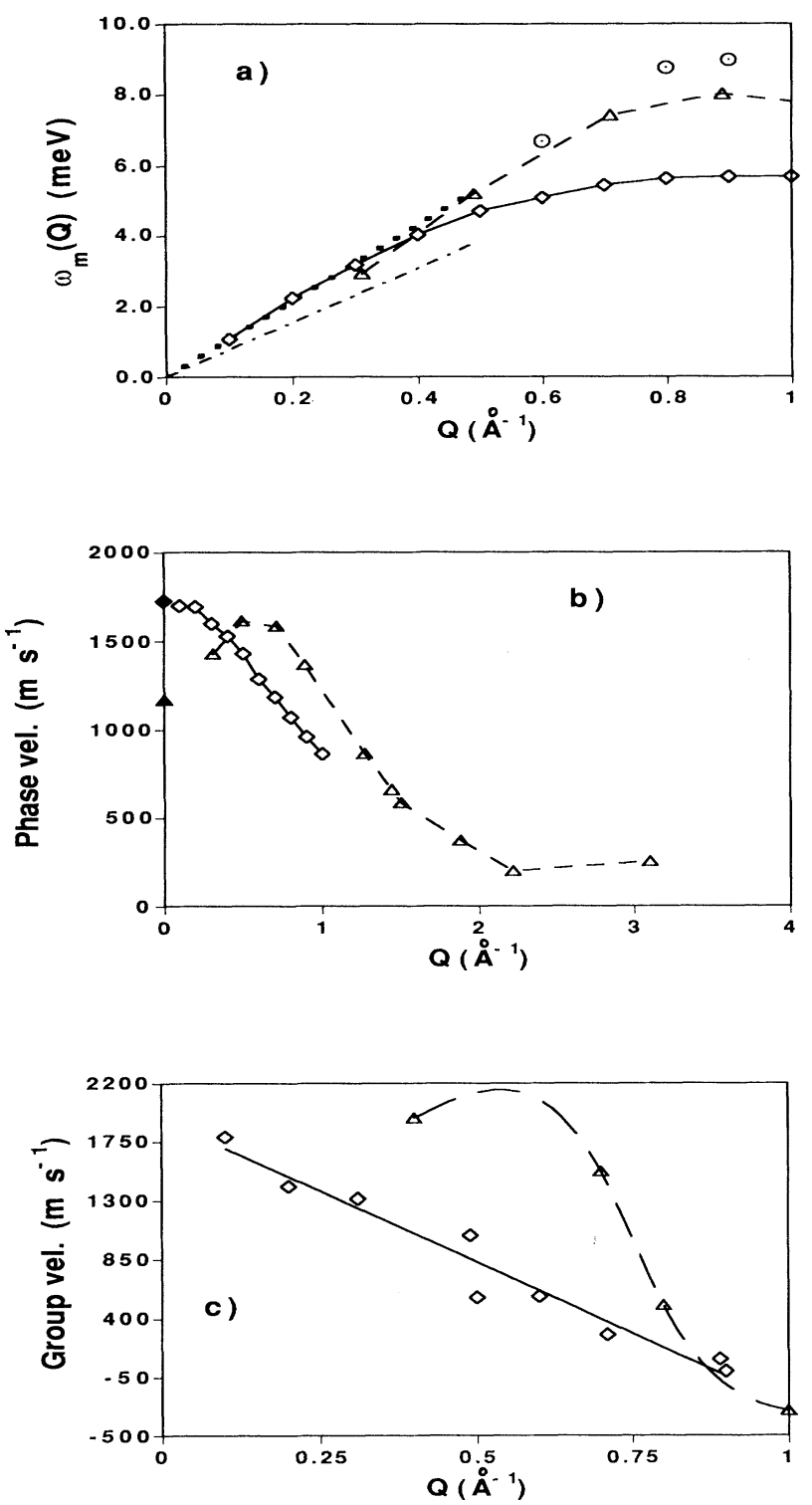

FIG. 7. (a) Frequencies corresponding to the maxima of the $J_{l}(Q, E)$ functions. The solid line with lozenges shows the polycrystalline data and the dashed line with triangles the MD glass. The open circles show the experimental points. The hydrodynamic sound velocities are indicated by dots (crystal) and dash-dots (glass). (b) Phase velocity dispersion for LD and MD data. The same symbols as above are used. The data at $Q=0$ give the hydrodynamic values quoted in the text. (c) Group velocity dispersion for LD and MD data. Same symbols are used. The crystal data are approximated by a straight line which leads to a value close to the hydrodynamic limit. The glass data are joined by a smooth curve which is drawn as a guide to the eye. comparison is shown in Fig. 8, where it can be seen how the square root of the $\mu_{2}(Q)$ reduced second frequency moments for the polycrystal approach the correct hydrodynamic limit up to relatively long wave vectors $Q_{c} \approx 0.4$ $\AA^{-1}$, whereas the MD and experimental points approach the long-wavelength value at $Q$ values near the lowest reachable by INS and MD $Q_{c} \leq 0.3 \AA^{-1}$, and therefore, the system-size limitations in the MD case and the kinematical restrictions in the experiment preclude any definite conclusion about this topic. The agreement between the values for the $\mu_{2}(Q)$ reduced moments for the experiment and $\mathrm{MD}$ results is rather remarkable if the moderate resolution employed in the present experiment is taken into account. The value of $Q_{c}$ can be used to estimate the smallest wavelength for acoustic excitations still being of extended (nonlocalized) nature. An estimate can then be obtained from $\lambda \approx 2 \pi / Q_{c}=21 \AA$, a value which is comparable to those calculated as phonon mean free paths within several frameworks. ${ }^{4}$

The interesting point to note regarding Fig. 8 is the
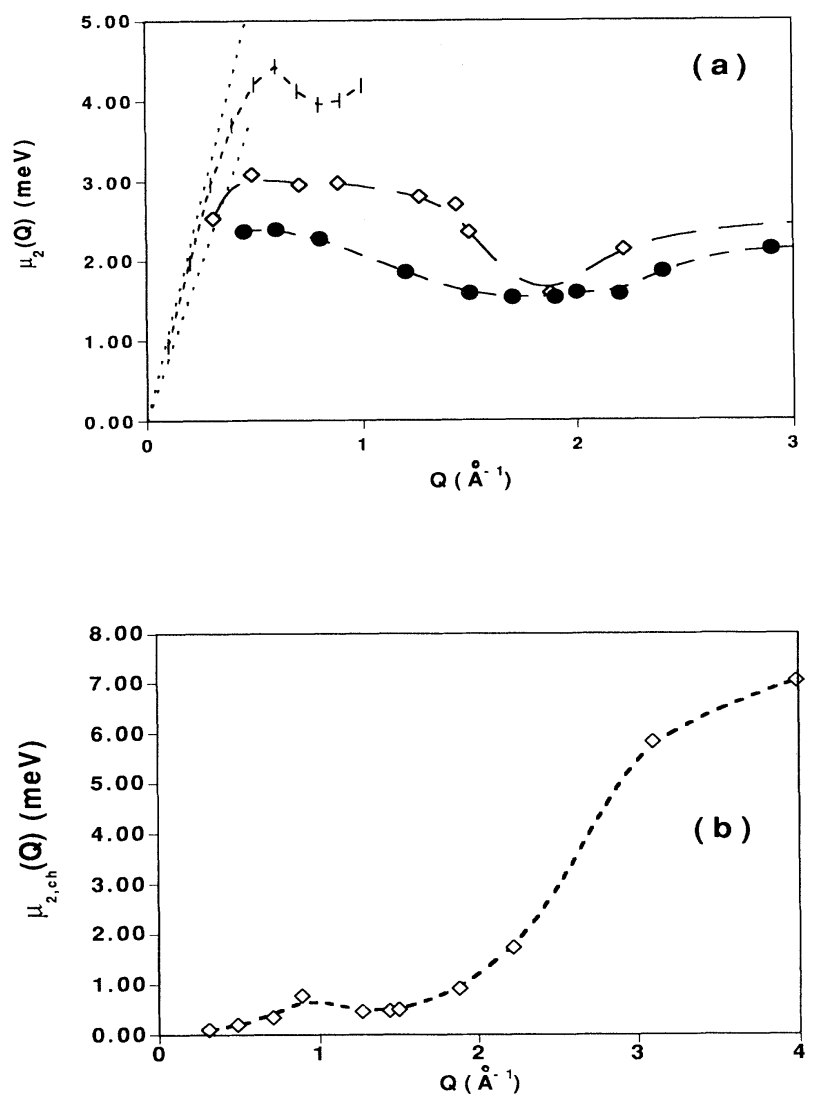

FIG. 8. The upper frame shows the experimental (filled circles) and calculated (open lozenges) $\mu_{2}(Q)$ (i.e., the square root of the normalized second frequency moment) for the glass, computed using a cutoff value of $E_{c}=6 \mathrm{meV}$ for the frequency integrals. The hydrodynamic sound velocity is shown by the dashed-space line. The vertical bars and the dotted line show the results for the polycrystal as calculated from the LD computation. (b) The reduced $\mu_{2, \mathrm{ch}}(Q)$ second frequency moment for an individual chain of selenium atoms. 
close value of the $\mu_{2}(Q)$ energies with that corresponding to the peak maxima of the low-frequency feature found in the glass spectra using higher-energy resolution. ${ }^{4}$ Furthermore, the peak position and shape of such a characteristic feature can be accounted for approximately if the values of $\mu_{2}^{2}(Q)$ and $\mu_{4}^{2}(Q)$ are used to reconstruct the line shape following the lines described in Ref. 22. Apart from the differences in excitation energies when compared with the curve of the total functions, some qualifications can be made regarding the oscillations exhibited by these single-chain quantities. First and foremost, no phase relationship exists with the total $S(Q)$ structure factor. The $\mu_{2, \mathrm{ch}}(Q)$ curve shows a concave curvature up to about $Q=1 \AA^{-1}$, then leading to a shallow minimum up to about $1.5 \AA^{-1}$, and then a strong increase in energies follows. In counterposition, the moments corresponding to the total function show a convex increase in frequencies up to about $Q_{p} / 2$ and then, as expected, a relative minimum at $Q_{p}$. Secondly, both the steep increase in energies above $1.5 \AA^{-1}$ and the calculated $S_{\text {inel }}(Q)$ inelastic (integrated) intensities indicate that above such momentum transfers the dynamics becomes increasingly dominated by intrachain motions. As a matter of fact, the spectra for the total sample and a single chain show no noticeable differences above $Q \approx 3$ $\AA^{-1}$.

To provide a comparison of the results commented on above, which are cast in terms of the spectral moments with those couched in terms of model parameters, the wave-vector dependence of the $E_{i}$ excitation energies derived from the analysis of the line shapes as well as the $\Gamma_{i}$ damping factors for the two oscillators used to represent the line shape are shown in Fig. 9, as well as with the frequency $\omega_{m}(Q)$ which corresponds to the maximum of the $J_{l}(Q, E)$ autocorrelation calculated from the fitted functions. As can be seen from the graph, the frequencies of the two oscillator functions are a relatively well-separated exception made of the region of momentum transfers located about $Q_{p}$. On the other hand, due to both statistical inaccuracies and ill conditioning of the fitting problem, no consistent dependence with the wave vectors was found for $E_{1}, \Gamma_{1}$, excitation energy, and damping of the low-frequency peak in both MD and experiment, and therefore only the $E_{1}$ values derived from fits to the MD spectra are plotted, as indicated in the figure. The $\omega_{m}(Q)$ showing the maxima of the total $J_{l}(Q, E)$ function and the $E_{2}$ curves which correspond to the excitation energies of the broad, more intense oscillator, can be nearly superimposed to that drawn for the $\mu_{2}(Q)$ moment of the scattering law shown in Fig. 8. The experimental data also shown in the figure are in tolerable agreement with the simulation up to $Q=1.2$ $\AA^{-1}$, if consideration is made of the moderate resolution . The data point at $Q=1.5 \AA^{-1}$ represents a particularly difficult case since the frequencies of the two oscillators become rather close.

A remarkable dependence with the wave vectors was also found for the damping terms as exemplified in Fig. 9. Nearly a quadratic dependence with momentumtransfers was found for $Q$ values up to about $1 \AA^{-1}$ regarding the damping term characteristic of the broad inelastic response. A deep minimum was also found for both the damping and the $\omega_{2}$ bare frequency, and beyond such momentum transfer the dominant contribution to $E_{2}$ comes from the damping terms. The parabolic dependence of the damping terms at low wave vectors as well as the presence of a well-defined maximum was also noticed in the analysis of the spectral response of another glass, ${ }^{23,24}$ although no consistent explanation for this behaviour seems to be presently available. ${ }^{25}$

The difficulty in analyzing the damping coefficients lies in the fact that a substantial part of the broadening does not arise from finite-lifetime effects (i.e., "homogeneous broadening") but has its origin on the continuum of frequencies brought about by the topological disorder of
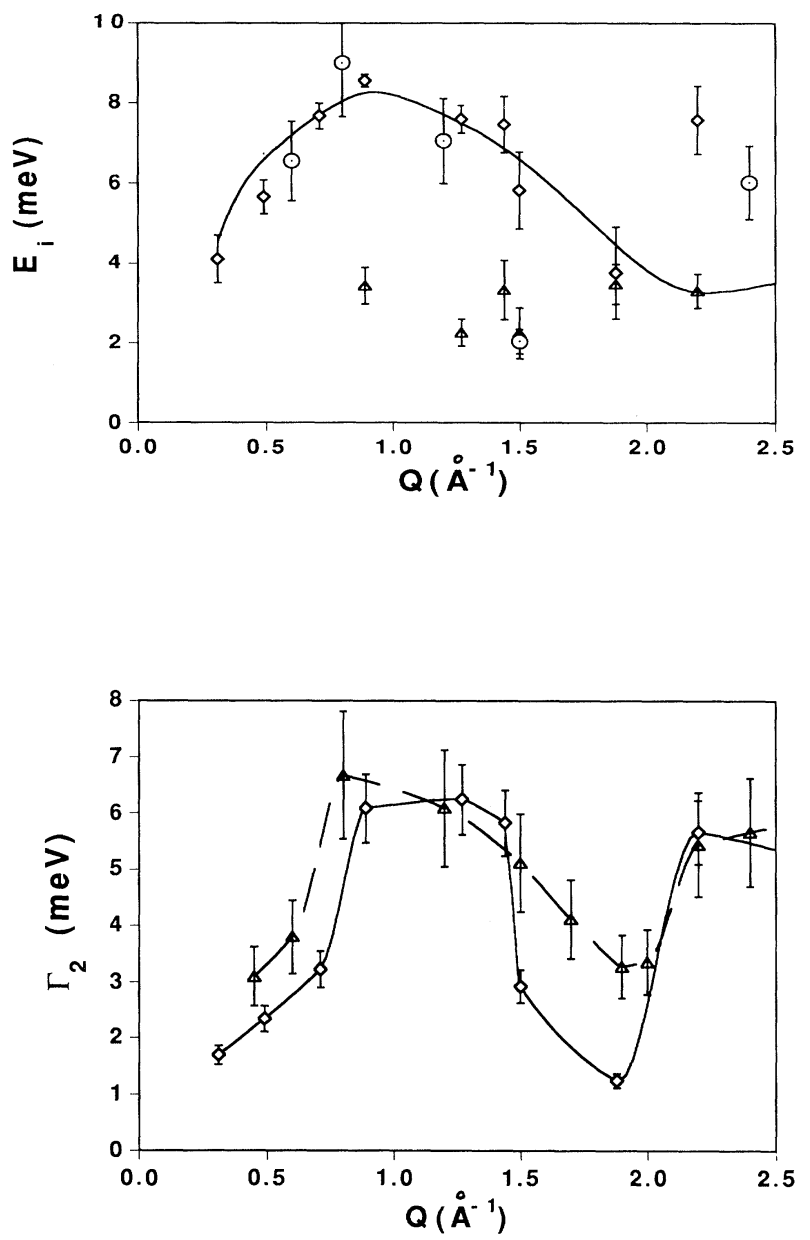

FIG. 9. The upper frame shows the $E_{i}$ excitation energies for the two oscillator functions as derived from the analysis of the line shapes of the MD (open lozenges and triangles) and experimental (open circles) spectra. The $\omega_{m}(Q)$ curve giving the wave-vector dependence of the maxima of the $J_{l}(Q, E)$ functions is shown by the solid line. The lower frame shows the $\Gamma_{2}$ damping factors corresponding to the higher-energy oscillator (see text). Open lozenges correspond to the analysis of MD data and triangles are estimates from the experimental spectra. The solid and dashed lines have been drawn as guides to the eye. 
the glass (i.e., "inhomogeneous broadening"). Up to the present moment there seems to be no adequate means to evaluate the latter effect.

In summary it seems clear that both approaches (i.e., in terms of spectral moments or from a model scattering law) to analyze the spectral functions lead to rather similar results, something which, on the other hand, enables the interpretation of the $\mu_{2}(Q)$ moments as physical (average) frequencies without having recourse to any detailed model function to represent the line shape. The agreement between the frequencies shown by the $\omega_{m}(Q)$ curves and the $E_{i}$ excitation frequencies, far from being fortuitous, is a consequence of the model used to represent the oscillator functions, ${ }^{16}$ which assigns a physical meaning to the $E_{i}$ quantities but not to their bare counterparts. In this latter respect it is worth emphasizing ${ }^{21}$ that only the $E_{i}$ frequencies are amenable of experimental measurement under moderate or heavy damping regimes.

\section{DISCUSSION AND CONCLUSIONS}

From the analysis of the Debye-Waller factors corresponding to an individual selenium chain as well as to the total sample, from the reconstruction of the lowfrequency part of the spectra from the c.m. part of the $Z(E)$ vibrational density of states, its contribution to the total heat capacity at low temperatures, and from the wave-vector dependences of the inelastic intensities and frequency moments, it seems clear that the dynamical response characteristic of the glassy state of selenium mostly arises from interchain interactions. The idea that most of the thermal anomalies characteristic of its glassy state were originated by a distinctive arrangement of the interactions between neighboring chains was advanced by Lucovsky, ${ }^{3}$ after careful intercomparison of experimental data from widely varying sources.

The value for the mean squared displacement derived from the $f_{\text {ch }}(Q)$ function $r_{s} \approx 0.02 \AA^{2}$ has been found to be very close to that obtained from experimental means, something which can indicate that most of the processes which are relevant to account for the motional amplitudes observed within the energy window characteristic of highresolution INS spectra arise from single-chain motions. The small value for the associated $M_{\text {eff }}$ effective mass of about 2.5 selenium atoms is also indicative of the rather localized character of the motions which contribute to this dynamical parameter. Most of the motional components entering this amplitude arise from excitations of acoustic and low lying optic origin, and the contribution of excitations below $2 \mathrm{meV}$ ( $\approx 500 \mathrm{GHz}$, a boundary used in Ref. 20 to derive a tentative scaling law) still represents about $60 \%$ of the total amplitude.

Although the physical soundness of a model of glassy selenium based upon relatively short chains can be put into question from a macroscopic point of view, recent results using chains of 40 atoms indicate that the most noticeable effect of increasing the chain length concerns the long-time properties of the high-temperature (liquid) phases associated with long-range (diffusive) movements as well as with the configurational statistics of a single chain. Such a finding agrees with recent results on the dynamic response of a Lennard-Jones chain, ${ }^{22}$ where it was found that the thermodynamic limit was approached by chains with $30-40$ atoms.

The analysis in terms of either the spectral moments or any other average frequency chosen for the purpose has shown that the microscopic dynamics of glassy selenium at time and length scales accessible to INS is extremely intricate due to the strong interactions between all the degrees of freedom, and cannot be sensibly understood in oversimple terms. As found for other molecular glass, ${ }^{7,23,26}$ the manifold of excitations of optical origin becomes, within the equivalent first Brillouin zone, far more important in the glass than in the polycrystal. As a matter of fact, and for momentum transfers as low as $0.4 \AA^{-1}$, such excitations will lead to a pullout of the average frequencies well above those characteristic of sound propagation.

Some comments are in order regarding the redistribution of spectral power in passing from the harmonic, orientationally averaged crystal, to the glass. First and foremost, the strong increase in "excess modes" in the low-frequency tails of the glass spectra, a phenomenon widely regarded as one of the fingerprints of the glassy state, is accompanied by shifts to lower frequencies of most of the peaks which are still identifiable in the glass spectra. On the other hand, the high-frequency bondstretching band contour shows a complicated trend. The two well-separated peaks seen in the polycrystal are still visible in the glass spectra, although in this case the center of gravity of such a band is displaced about $1 \mathrm{meV}$ towards higher energies, a fact which is also borne out from the experimental data. ${ }^{11}$ Also, as illustrated in some detail in the preceding section, below the zone boundary the spectra of the glass show a rather intricate structure over a wide extent in frequencies, in counterposition to the crystal where a strong peak, signature of sound propagation, is visible up to $Q \approx 1 \AA^{-1}$.

As a final conclusion, what seems to be common for glassy selenium and other vitreous materials studied following a similar approach, ${ }^{7,23,8}$ is the fact that, disregarding the widely varying chemical complexity, the internal modes of the glass-forming entities, whether they are simple molecular units or polymerlike atomic assemblies, are not the major contributors to the part of the frequency spectrum responsible for the thermal anomalies, since their manifestation can be accounted for, at least on a semiquantitative basis, using the center-of-mass quantities.

\section{ACKNOWLEDGMENTS}

This work has been supported in part by DGICYT Grant No. PB89-0037-C03. Very helpful discussions with Professor U. Buchenau and J. Alonso are kindly acknowledged. 
${ }^{1}$ See, for instance, The Physics of Selenium and Tellurium, edited by E. Gerlach and P. Grosse, Vol. 13 of Springer Series in Solid State Sciences (Springer, Berlin, 1979).

${ }^{2}$ W.A. Phillips, U. Buchenau, N. Nücker, A.J. Dianoux, and W. Petry, Phys. Rev. Lett. 63, 2381 (1989).

${ }^{3}$ In The Physics of Selenium and Tellurium (Ref. 1), p. 178. A detailed discussion on the bonding structures present in the liquid and glassy phases is also presented. See also G. Lucovsky and C.K. Wong, J. Non-Cryst. Solids 75, 51 (1985).

${ }^{4}$ For a phenomenological approach based upon the soft-mode concept, see U. Buchenau, Yu M. Galperin, V.L. Gurevich, D.A. Parshin, M.A. Ramos, and H. Schober, Phys. Rev. B 46, 2798 (1992). An extension of this treatment to undercooled liquids is given in U. Buchenau, J. Mol. Struct (to be published).

${ }^{5}$ S.R. Nagel, A. Rahman, and G.S. Grest, Phys. Rev. Lett. 53, 368 (1984).

${ }^{6}$ H.R. Schober and B.B. Laird, Phys. Rev. B 44, 6746 (1991).

${ }^{7}$ F.J. Bermejo, J. Alonso, A. Criado, F.J. Mompeán, J.L. Martinez, M. García-Hernández, and A. Chahid, Phys. Rev. B 46, 6173 (1992).

${ }^{8}$ M. García-Hernández, R. Burriel, F.J. Bermejo, C. Piqué, and J.L. Martinez, J. Phys: Condens. Matter 4, 9581 (1992).

${ }^{9}$ N.G. Almarza, E. Enciso, and F.J. Bermejo, Europhys. Lett. 17, 595 (1992).

${ }^{10}$ M. García-Hernández, F.J. Bermejo, B. Fåk, J.L. Martinez, E. Enciso, N.G. Almarza, and A. Criado, Phys. Lett. A 175, 217 (1993).

${ }^{11}$ See F. Gompf, in The Physics of Selenium and Tellurium (Ref. 1), p. 64; G. Carini, M. Cutroni, A.J. Dianoux, G. Galli, L.M. Needham, and H.M. Rosenberg, J. Non-Cryst. Solids 117-118, 140 (1990).

${ }^{12} \mathrm{D}$. Hohl and R. Jones, Phys. Rev. B 43, 3856 (1991).

${ }^{13}$ The lowest frequencies reachable in our simulation are about $0.6 \mathrm{meV}$, something which is enough to cover the most prominent low-frequency peaks in $Z(E)$, centered at about $1 \mathrm{THz}(4 \mathrm{meV})$.

${ }^{14} \mathrm{~W}$. Gotze, in Liquids, Freezing and the Glass Transition, Proceedings of the Les Houches Summer School of Theoretical Physics Session LI, edited by J.P. Hansen et al.
(North-Holland, Amsterdam, 1991), p. 287.

${ }^{15} \mathrm{H}$. Wendel, in The Physics of Selenium and Tellurium (Ref. 1), p. 47. See also R.M. Martin, G. Lucovsky, and K. Helliwell, Phys. Rev. B 13, 1383 (1976).

${ }^{16}$ The functional form for the damped harmonic oscillator is discussed in B. Fåk and B. Dorner (unpublished).

${ }^{17}$ U. Buchenau, Z. Phys. 58, 181 (1985).

${ }^{18}$ L.D. Landau and E.M. Lifshitz, Statistical Physics (Pergamon, Oxford, 1987), Chap. 12.

${ }^{19}$ R. Grosse, P. Krause, M. Meissner, and A. Tausend, J. Phys: Solid State 11, 45 (1978), and references therein. Also, M. Meissner and D. Wobig, in The Physics of Selenium and Tellurium (Ref. 1), p. 68; S.S. Chang and A.B. Bestul, J. Chem. Thermodyn. 6, 325 (1974). The values of the Debye velocities in the trigonal crystal have been taken from M. Meissner, in The Physics of Selenium and Tellurium (Ref. 1), p. 74 .

${ }^{20}$ U. Buchenau and R. Zorn, Europhys. Lett. 18, 523 (1992).

${ }^{21}$ The form used to represent the damped harmonic oscillator enables the identification of the $E_{i}$ renormalized excitation energies with the maxima of the $J_{l}(Q, E)$ autocorrelation functions.

${ }^{22}$ A.R. McGurn, A.A. Maradudin, and R.F. Wallis, in Microscopic Aspects of Non-linearity in Condensed Matter, Vol. 264 of NATO Advanced Study Institute, Series B: Physics, edited by A.R. Bishop et al. (Plenum, New York, 1991), p. 293; A. Cuccoli, V. Togneti, A.A. Maradudin, A.R. McGurn, and R. Vaia, Phys. Rev. B 46, 8839 (1992).

${ }^{23}$ F.J. Bermejo, J. Alonso, A. Criado, J.L. Martinez, and M. García-Hernández (unpublsihed).

${ }^{24}$ F.J. Bermejo, F. Batallan, D. Martin, J.L. Martinez, and M. García-Hernández, Phys. Lett. A 150, 250 (1990).

${ }^{25}$ To our knowledge, the only significant result concerning a strong dependence of the damping with the wave vectors is the one reported by W. Schirmacher and M. Wagener, in Dynamics of Disordered Materials, edited by D. Richter et al. (Springer-Verlag, Berlin, 1989), p. 231, which was based upon an effective medium approximation treatment.

${ }^{26}$ F.J. Bermejo, J.L. Martinez, D. Martin, and M. GarcíaHernández, Europhys. Lett. 15, 509 (1991).

${ }^{27}$ O. Brand and H. v. Löhneysen, Europhys. Lett. 16, 455 (1991). 